\title{
Comparison Study between Waypoints Tracking and Trajectory Tracking Control Strategies Using the Same PID Autopilot for Unmanned Helicopter Model
}

\begin{abstract}
A. Hosny*
Abstract: This paper is presenting a detailed comparison study for two different guidance strategies. The first strategy is concerning the difference angle between the desired heading or elevation referred to the next waypoint and the actual heading or elevation of the unmanned helicopter model. The second strategy is concerning the relative distance between the actual and the desired trajectories. In other words the first method is tracking the waypoints while the second one is tracking the trajectory. In this work a comparison study was conducted through the mentioned strategies simulation to show the significant differences in the output performance. Some performance indexes were presented to evaluate the system performance errors and the control effort needed for both strategies using the same desired trajectory and the same waypoints.
\end{abstract}

Keywords: UAH unmanned aerial helicopter, RC Remote Control, PPM Pole Placement Method, GA Genetic Algorithm, TTM Trajectory Tracking Method, WPTM Waypoint Tracking Method, Jerk, FFR Fixed Frame of Reference, BFR Body Frame of Reference.

\section{Guidance Approach}

\subsection{TTM Trajectory Tracking Method}

This approach is concerning the relative distances such as the lateral and the vertical distances with respect to the desired trajectory current segment DTCS body axis Fig. 1. In this method (in case of lateral control) inner and outer loops will be required, the inner loop will control the yaw rate and the outer loop will be fed by the lateral relative distance with respect to the desired trajectory current segment DTCS body axis while the feedback signal will represent the change in the heading as the absolute heading is not useful in this case. Both the inner and the out loop gains will be tuned by several trials after system integration. This approach leads to minimize the relative distances between the actual flight path and the desired trajectory.

\subsection{WPTM Waypoint Tracking Method}

This approach is concerning the relative heading and elevation with respect to waypoints Fig. 2. Applying some vector analysis the required change in heading or elevation will be easily calculated, consequently they will be fed to the yaw rate inner loop after tuning the command input gain according to the performance criteria, [1].

*PhD, Air Force R\&D, ahmed160771@yahoo.com 


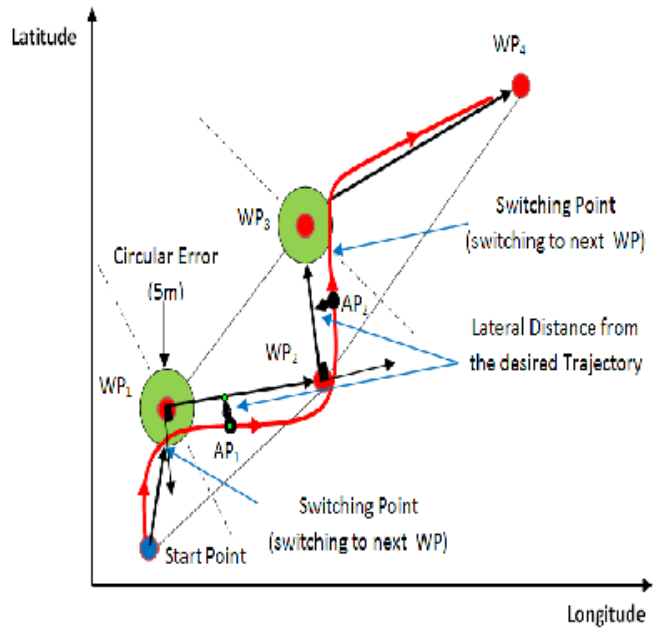

Fig. 1. Lateral Distances using Trajectory Tracking Strategy

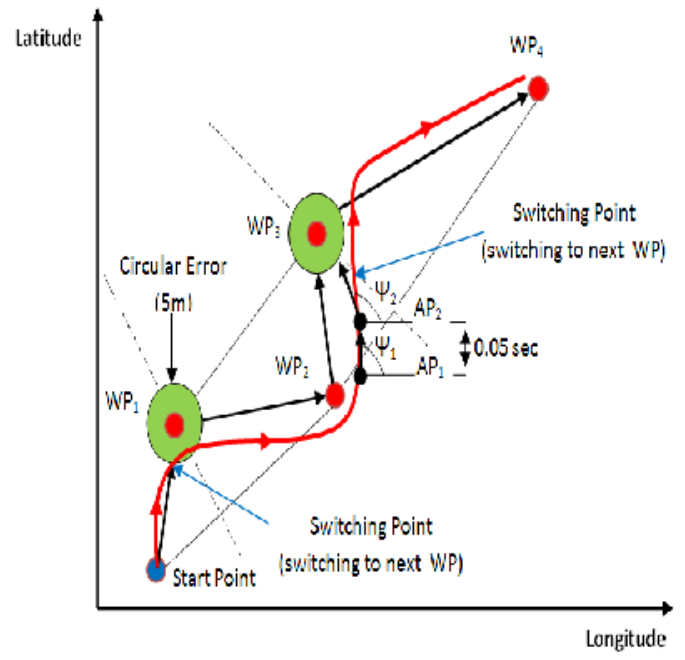

Fig. 2. Relative Heading using Waypoint Tracking Strategy

The following equations explain the transformation procedure starting from the actual flying model position with respect to fixed frame of reference to body coordinate of the DTCS with estimating a small initial conditions of (0.0001 for eg.) to avoid the singularities during simulation calculation Fig. 3, [2, 3].

$\mathrm{X}=$ Flying Model Actual Longitude (X component) relative to the fixed frame of reference $\mathrm{Y}=$ Flying Model Actual Latitude (Y component) relative to the fixed frame of reference $\mathrm{Z}$ = Flying Model Actual Altitude ( $\mathrm{Z}$ component) relative to the fixed frame of reference $X_{\mathrm{F}}=$ The Start Point of the Desired Trajectory Current Segment DTCS (X component) relative to the fixed frame of reference

$\mathrm{Y}_{\mathrm{F}}=$ The Start Point of the Desired Trajectory Current Segment DTCS (Y component) relative to the fixed frame of reference

$Z_{F}=$ The Start Point of the Desired Trajectory Current Segment DTCS ( $Z$ component) relative to the fixed frame of reference

$\mathrm{X}_{\mathrm{BO}}=$ Flying Model Actual Longitude (X component) relative to DTCS body axes at the fixed frame of reference

$\mathrm{Y}_{\mathrm{BO}}=$ Flying Model Actual Latitude (Y component) relative to DTCS body axes at the fixed frame of reference

$\mathrm{Z}_{\mathrm{BO}}=$ Flying Model Actual Altitude ( $\mathrm{Z}$ component) relative to DTCS body axes at the fixed frame of reference

$\mathrm{X}_{\mathrm{B}}=$ Flying Model Actual Longitude (X component) relative to DTCS body axes

$\mathrm{Y}_{\mathrm{B}}=$ Flying Model Actual Latitude (Y component relative) to DTCS $\mathrm{t}$ body axes

$\mathrm{Z}_{\mathrm{B}}=$ Flying Model Actual Altitude ( $\mathrm{Z}$ component) relative to DTCS body axes

$\theta, \quad, \Psi=$ Rotation around $\mathrm{X}, \mathrm{Y}, \mathrm{Z}$ axes
$\mathrm{A}=\cos \theta \cos \psi$
$\mathrm{B}=(\sin \sin \theta \cos \psi-\cos \quad \sin \psi)$
$\mathrm{C}=(\cos \sin \theta \cos \psi+\sin \quad \sin \psi)$
$\mathrm{D}=\cos \theta \sin \psi$
$\mathrm{E}=\left(\begin{array}{lll}\sin & \sin \theta \sin \psi+\cos \quad \cos \psi\end{array}\right)$
$\mathrm{F}=\left(\begin{array}{lll}\cos & \sin \theta \sin \psi-\sin \quad \cos \psi\end{array}\right)$ 


$$
\begin{aligned}
& \mathrm{G}=-\sin \theta \\
& \mathrm{M}=\sin \cos \theta \\
& \mathrm{N}=\cos \cos \theta \\
& Z_{B O}=\frac{[(Y A-X D)(B G-M A)-(X G-Z A)(E A-B D)]}{[(F A-C D)(B G-M A)-(C G-N A)(E A-B D)]} \\
& Y_{B O}=\frac{\left[(Y A-X D)-Z_{B O}(F A-C D)\right]}{(E A-B D)} \\
& X_{B O}=\frac{\left(X-Y_{B O} B-Z_{B O} C\right)}{A} \\
& Z_{\text {SHIFT }}=\frac{\left[\left(Y_{F} A-X_{F} D\right)(B G-M A)-\left(X_{F} G-Z_{F} A\right)(E A-B D)\right]}{[(F A-C D)(B G-M A)-(C G-N A)(E A-B D)]} \\
& Y_{\text {SHIFT }}=\frac{\left[\left(Y_{F} A-X_{F} D\right)-Z_{S H I F T}(F A-C D)\right]}{(E A-B D)} \\
& X_{\text {SHIFT }}=\frac{\left(X_{F}-Y_{\text {SHIFT }} B-Z_{\text {SHIFT }} C\right)}{A} \\
& \mathrm{X}_{\mathrm{B}}=\mathrm{X}_{\mathrm{BO}}-\mathrm{X}_{\text {shift }} \\
& \mathrm{Y}_{\mathrm{B}}=\mathrm{Y}_{\mathrm{BO}}-\mathrm{Y}_{\text {shift }} \\
& \mathrm{Z}_{\mathrm{B}}=\mathrm{Z}_{\mathrm{BO}}-\mathrm{Z}_{\text {shift }}
\end{aligned}
$$

\section{Actual Flying Model Coordinates to DTCS Body Axis at the Fixed Frame of Reference Origin}

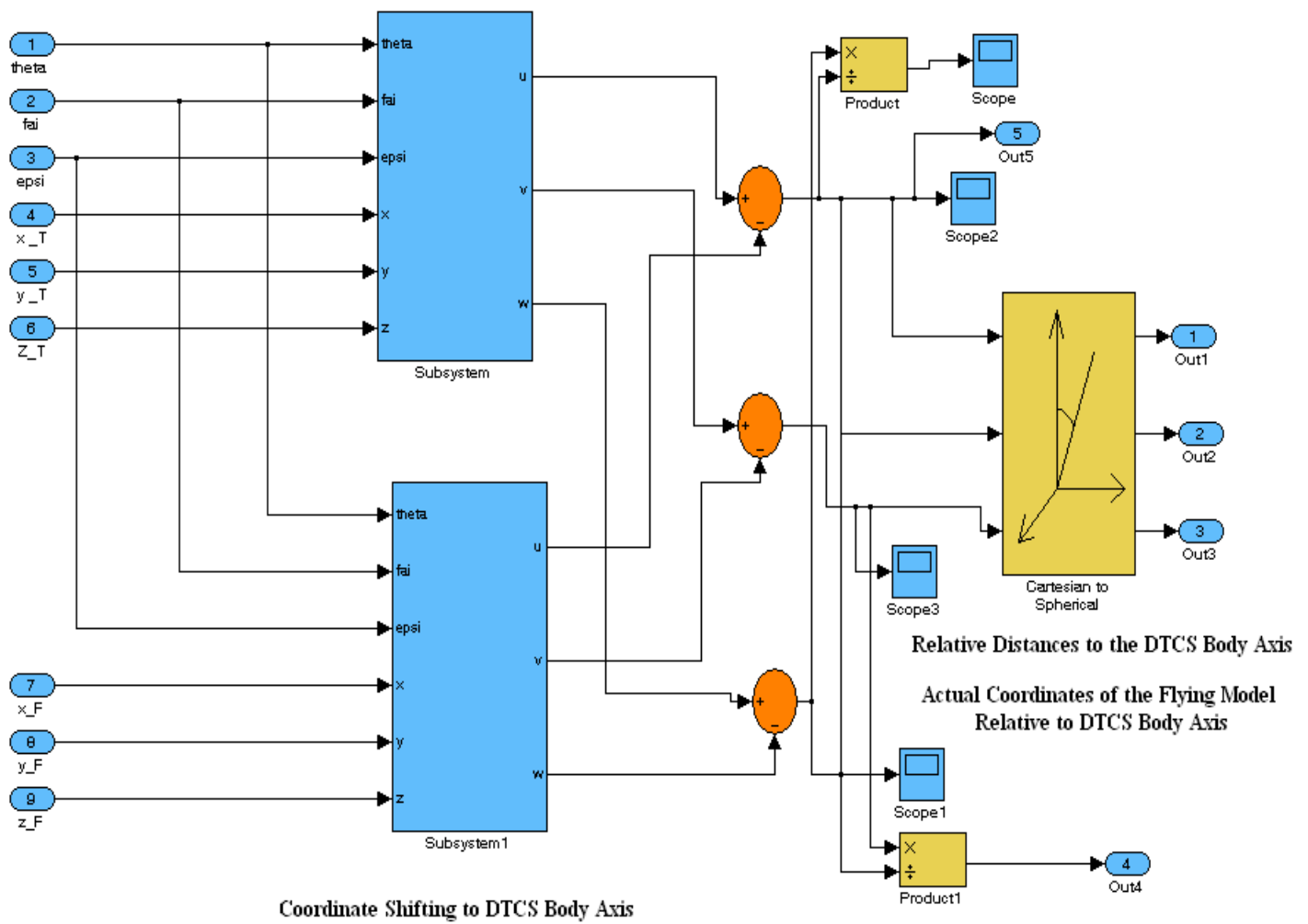

Fig. 3. Calculating the Flying Model Relative Distances With Respect to DTCS Body

\section{System Performance}

Axis

In the following figures Fig. 4 through Fig. 11 it is clear that the Trajectory Tracking Method TTM has a great and obvious impact in minimizing the relative distance between the actual 
flight path and the desired trajectory, the figures show that the flying model almost flying stick to the desired path when TTM is used while it tracks the waypoints only when WPTM is used regardless the relative distances between the actual and the desired path, thus increasing the relative distances (relative errors) when WPTM is used compared to the relative distances if TTM is used. Fig. 18 shows also a considerable fluctuation with large amplitude when TTM is used rather than when WPTM is used expressing a large control energy consumed during TTM due to the high number of attempts performed by the flying model to track each segment in the desired trajectory segments. Fig. 22 and Fig. 23 show that TTM would have more endurance than the WPTM for the same range (or mission), consequently TTM would have less range than WPTM for a given amount of fuel.

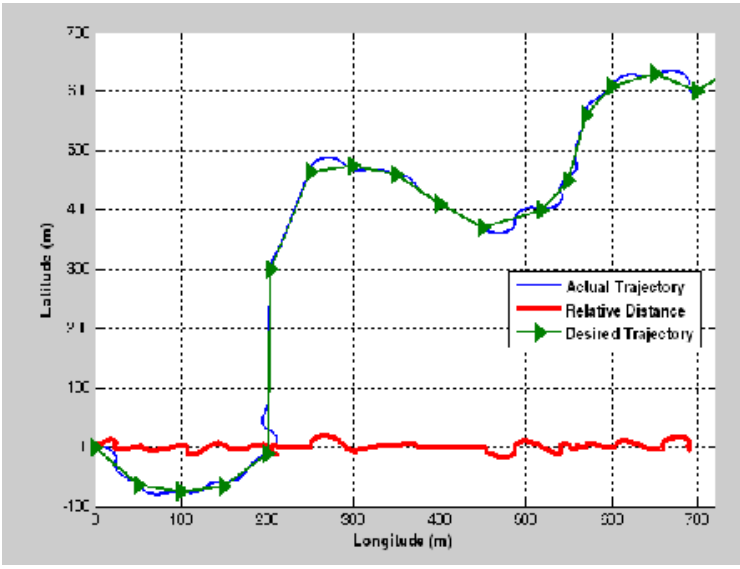

Fig. 4. Actual and Desired Trajectories in XY Plane with the Relative Lateral Distances (in DTCS body axis) For TTM

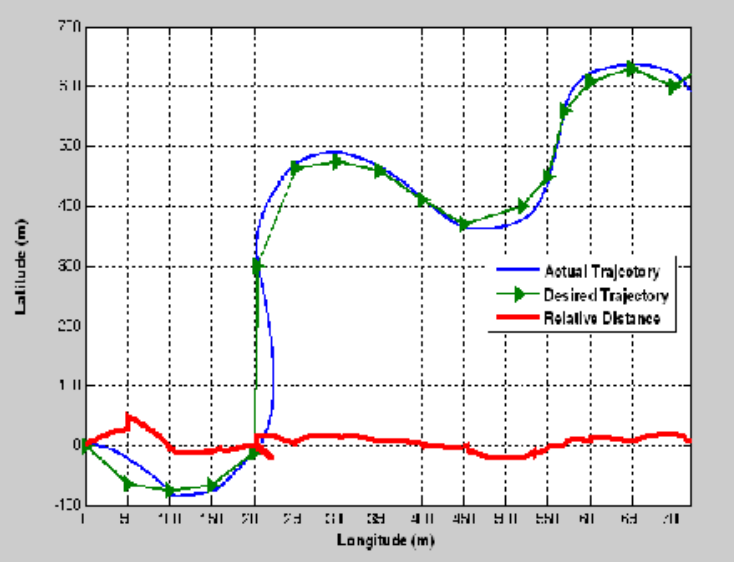

Fig. 5. Actual and Desired Trajectories in XY Plane with the Relative Lateral Distances (in DTCS body axis) for WPTM

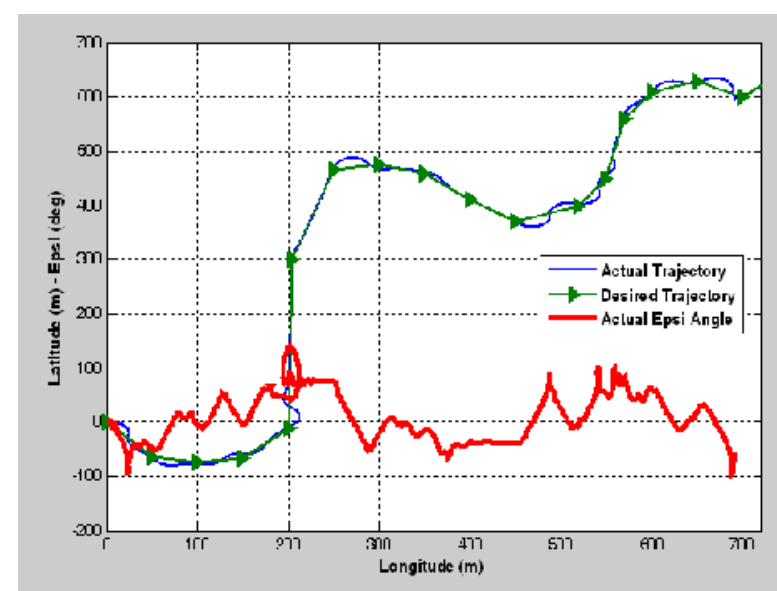

Fig. 6. Actual and Desired Trajectories with Actual Heading Angle For TTM

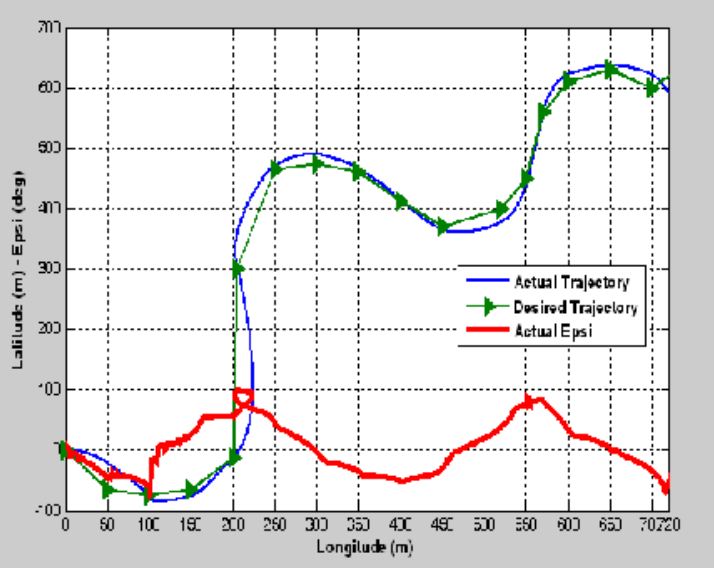

Fig. 7. Actual and Desired Trajectories with Actual Heading Angle For WPTM 


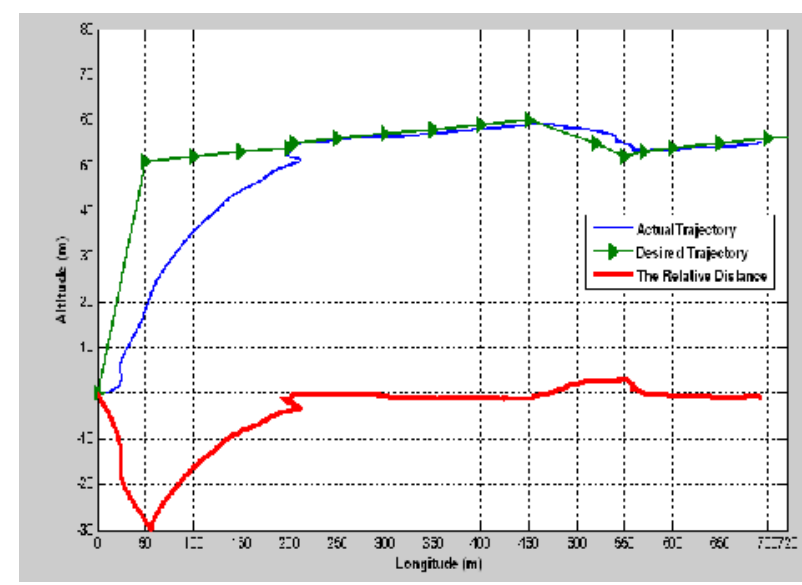

Fig. 8. Actual and Desired Trajectories in XZ Plane with the Relative Vertical Distances (in DTCS body axis) For TTM

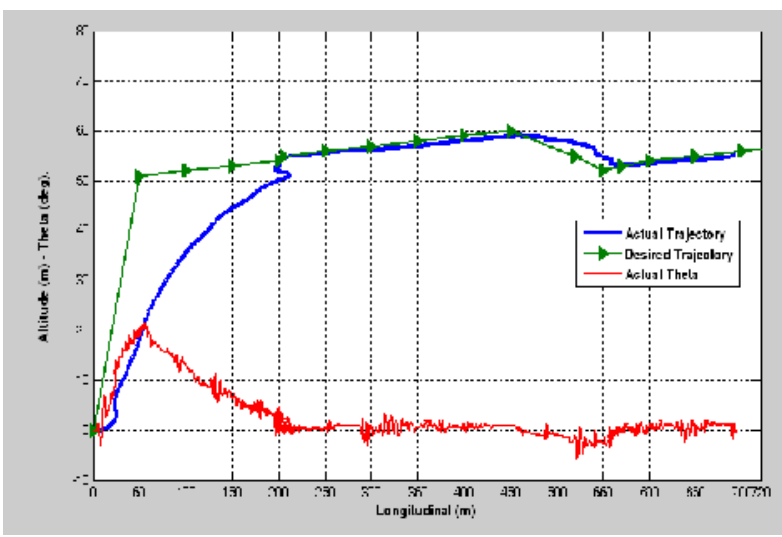

Fig. 10. Actual and Desired Trajectories in XZ Plane with Actual Elevation For TTM

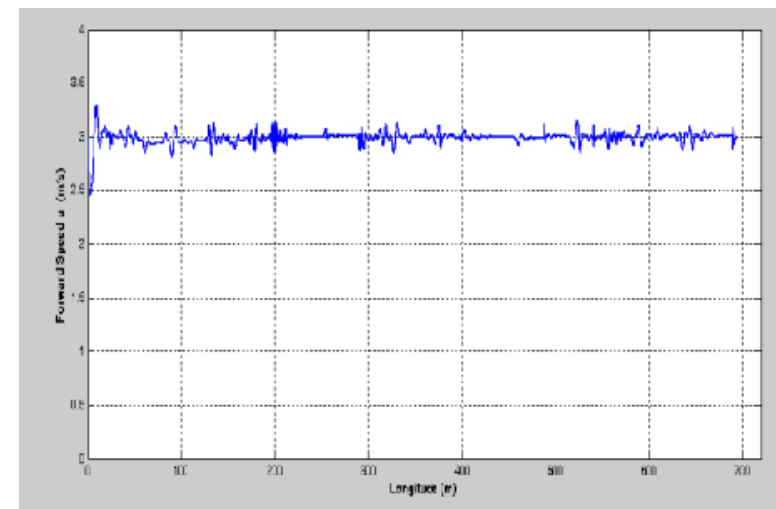

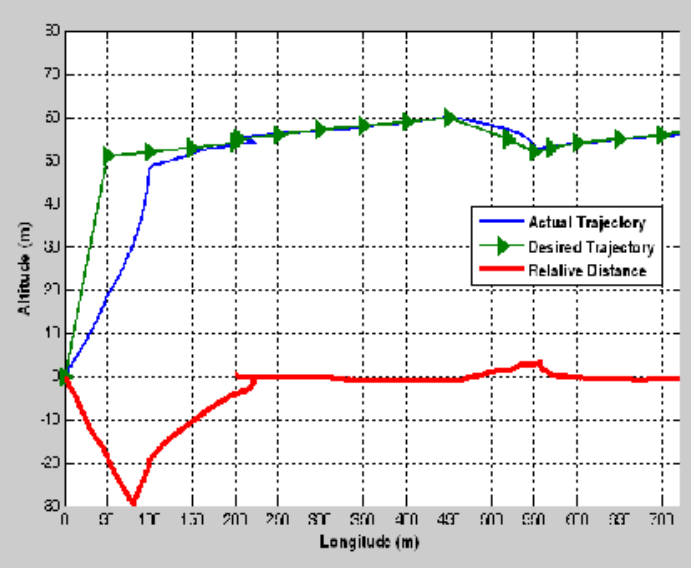

Fig. 9. Actual and Desired Trajectories in XZ Plane with the Relative Vertical Distances (in DTCS body axis) For WPTM

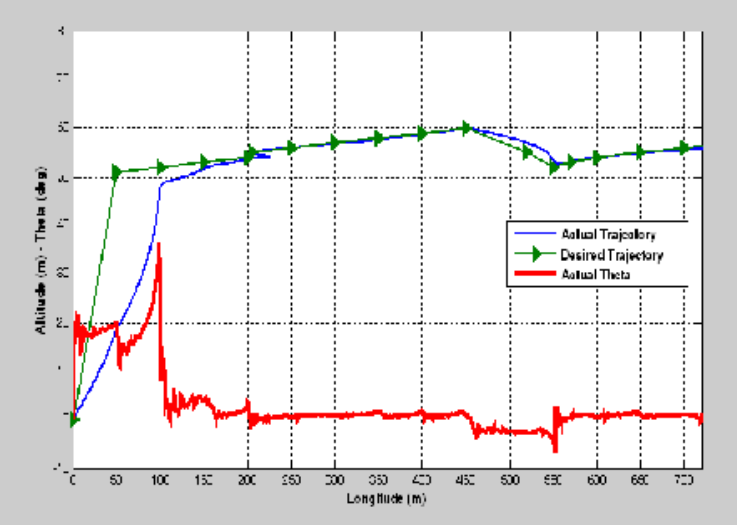

Fig. 11. Actual and Desired Trajectories in XZ Plane with Actual Elevation For WPTM

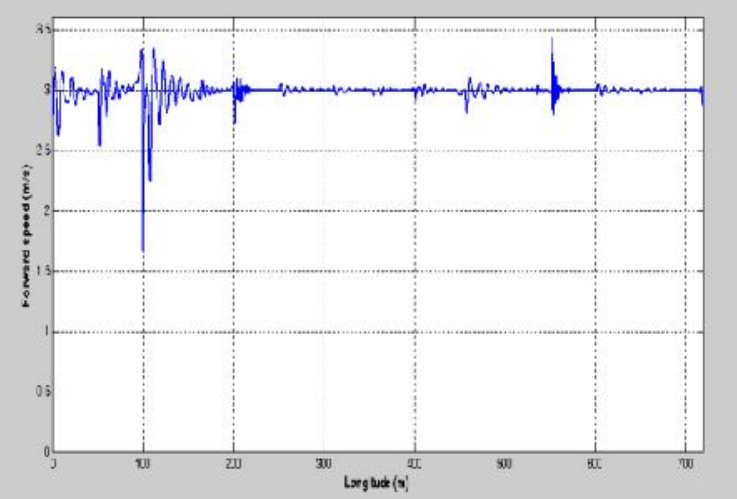

Fig. 12. Actual and Desired Forward Speed Fig. 13. Actual and Desired Forward Speed For TTM For WPTM 


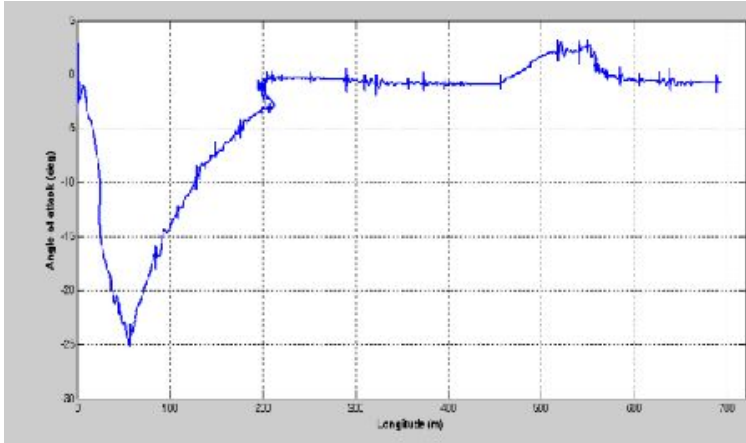

Fig. 14. Angle of Attack For TTM

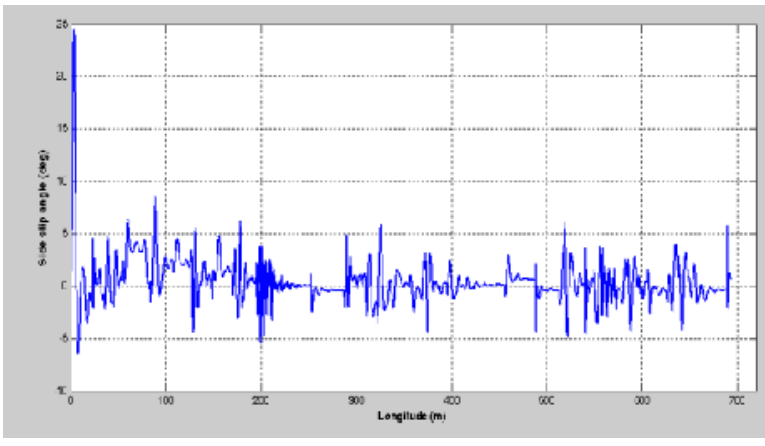

Fig. 16. Side Slip Angle For TTM

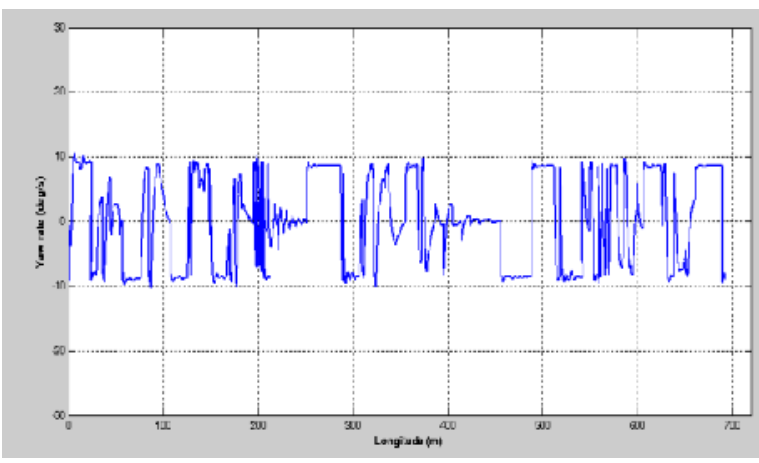

Fig. 18. Yaw Rate For TTM

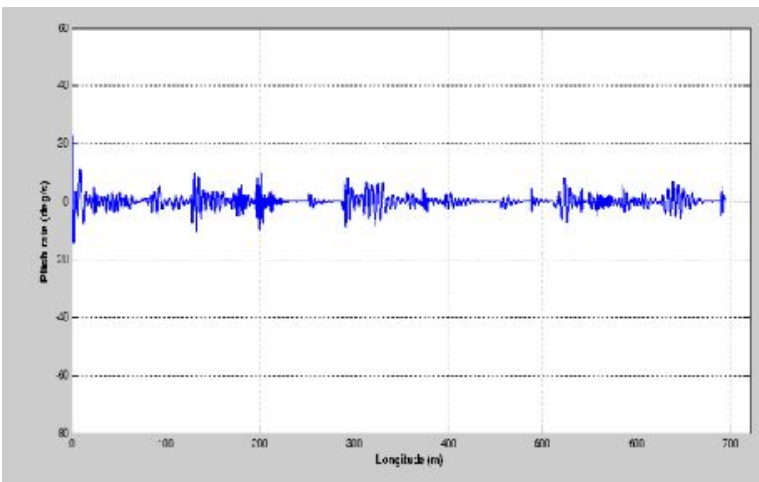

Fig. 20. Pitch Rate For TTM

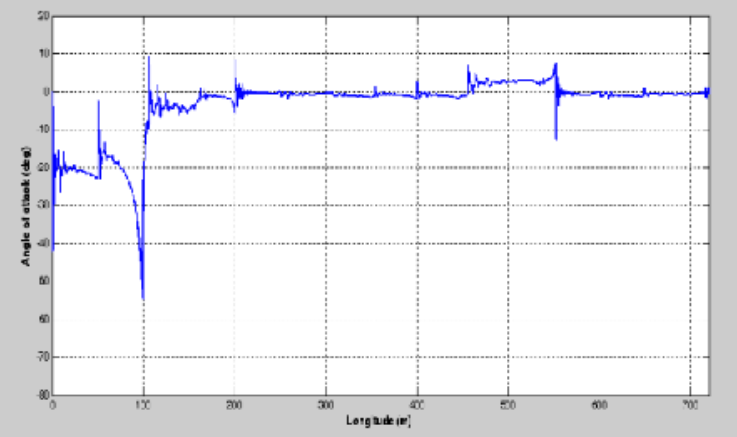

Fig. 15. Angle of Attack For WPTM

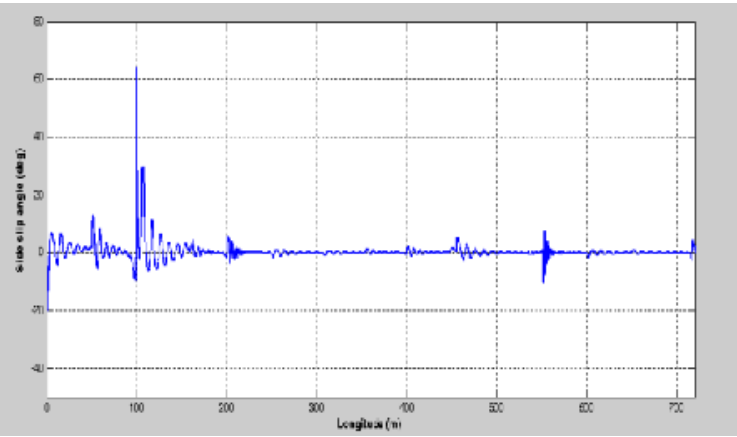

Fig. 17. Side Slip Angle For WPTM

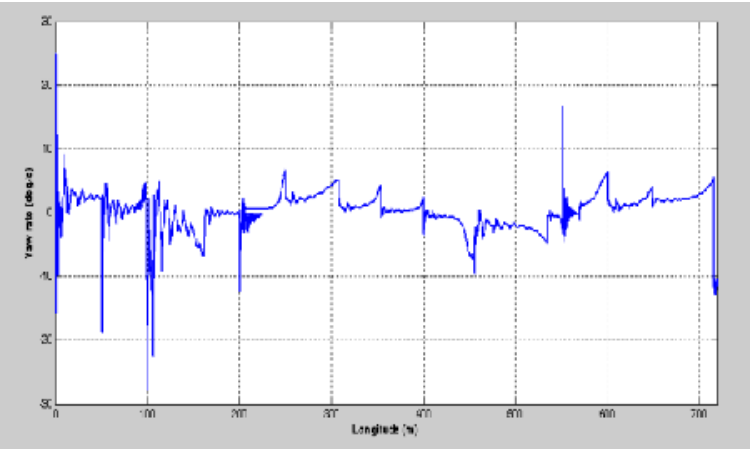

Fig. 19. Yaw Rate For WPTM

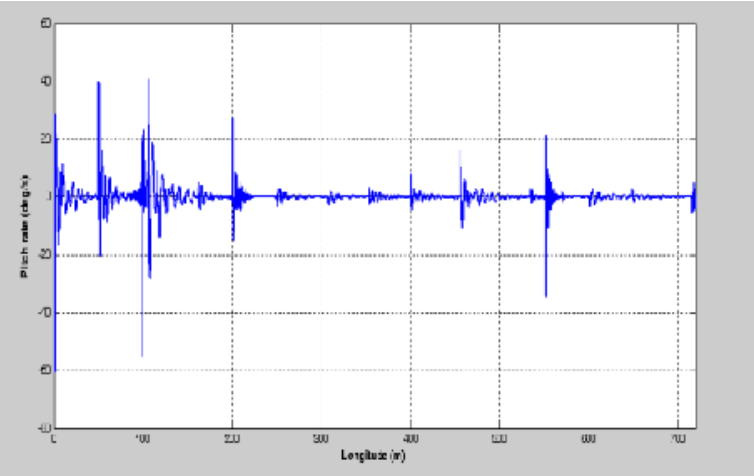

Fig. 21. Pitch Rate For WPTM 


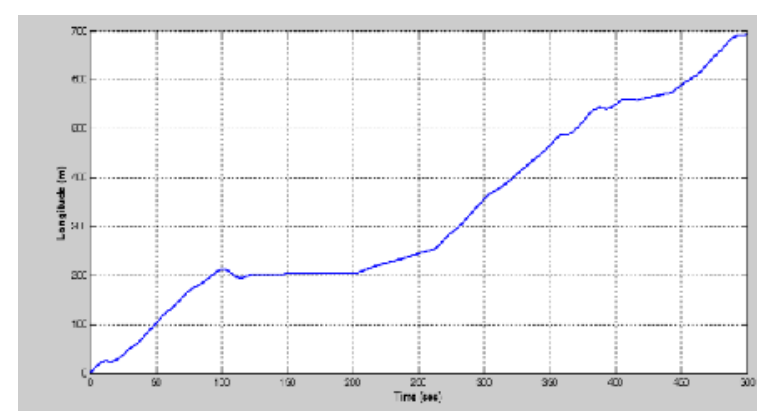

Fig. 22. Range and Endurance For TTM

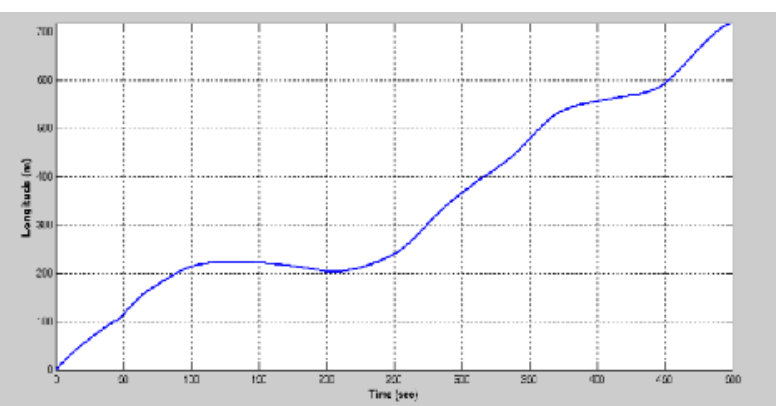

Fig. 23. Range and Endurance For WPTM

\section{Control System Performance}

Fig. 24 through Fig. 31 illustrate the control efforts done by each servo to perform the maneuvers required for the desired flight path. Fig. 30 shows an obvious fluctuation with large amplitude that would need a considerable amount of electric energy. This number of fluctuations would reduce the servos life time, and decrease the mean time between failures.

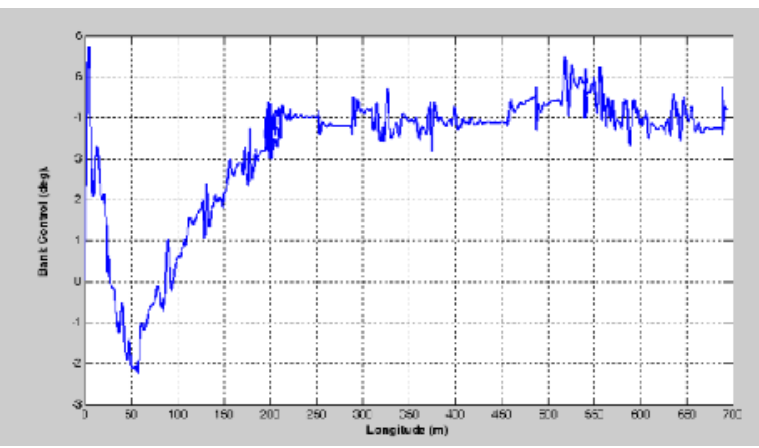

Fig. 24. Lateral Control Effort For TTM

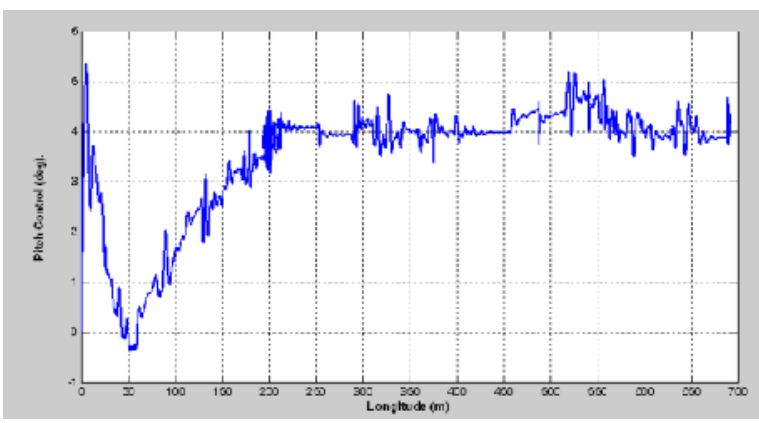

Fig. 26. Pitch Control Effort For TTM

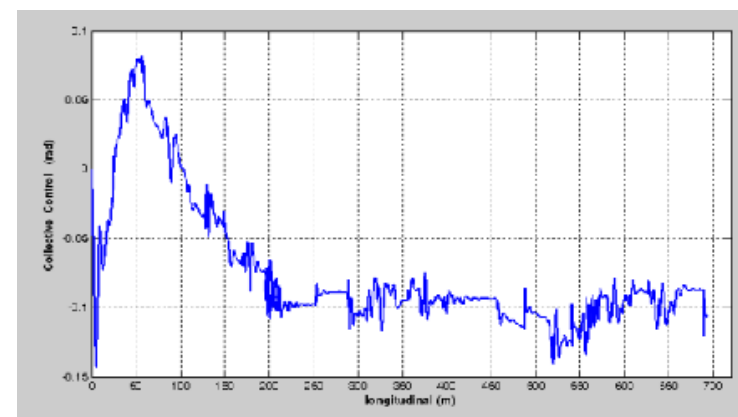

Fig. 28. Collective Control Effort For TTM

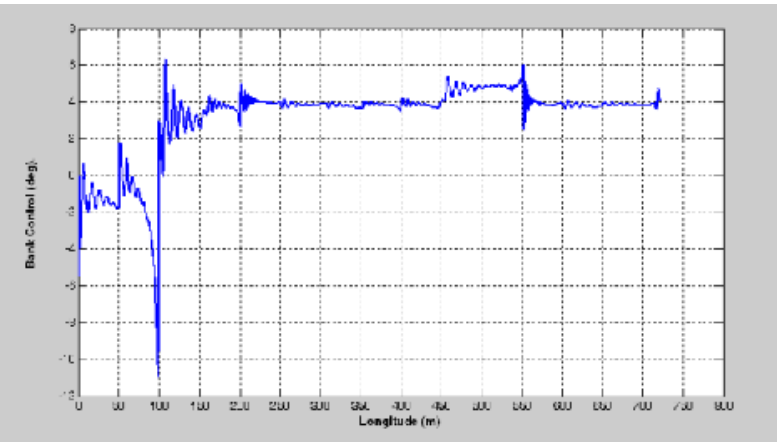

Fig. 25. Lateral Control Effort For WPTM

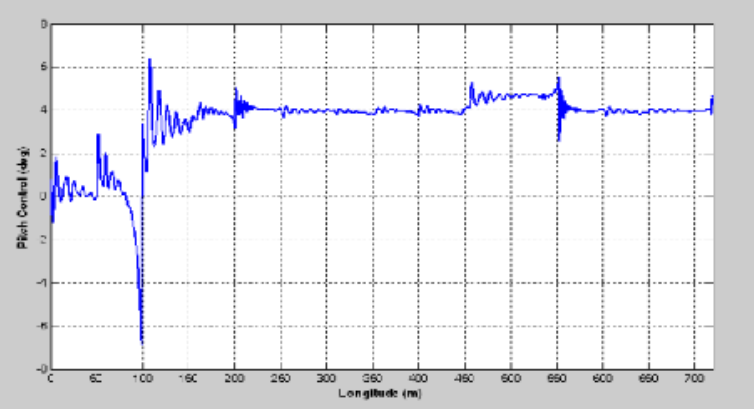

Fig. 27. Pitch Control Effort For WPTM

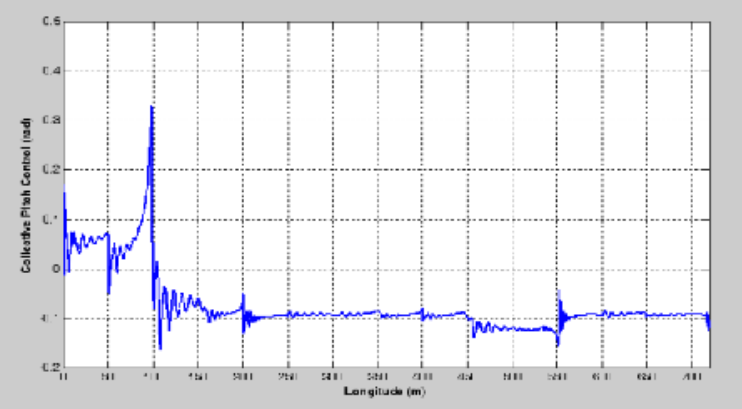

Fig. 29. Collective Control Effort For WPTM 


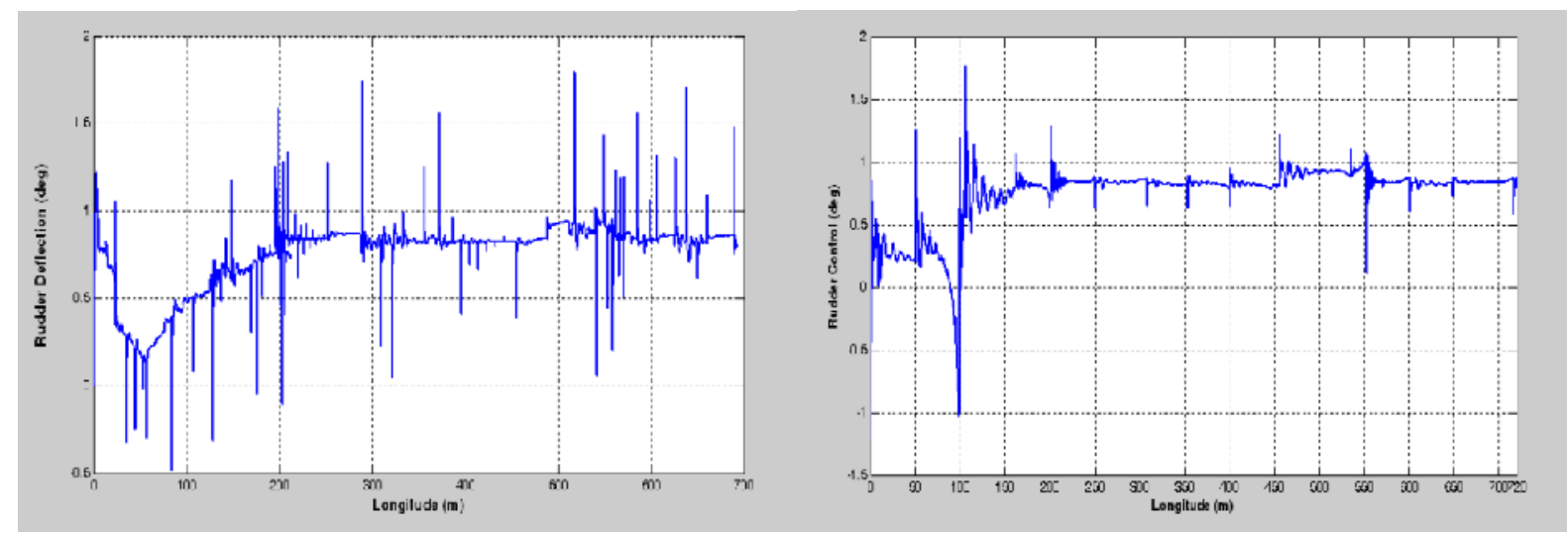

Fig. 30. Rudder Control Effort For TTM

Fig. 31. Rudder Control Effort For WPTM

\section{System Evaluation}

The integral absolute relative distance in both (X, Y) directions (in DTCS body axis) was chosen to be the evaluation criteria (Performance Index PI) in this work as it is shown in figures Fig.4 through Fig. 11. Figures Fig. 32 and Fig. 33 show that the Trajectory Tracking Method TTM has less (Performance Index PI) when it is compared with Waypoint Tracking Method WPTM though the (Performance Index PI) using WPTM is slightly less than when using TTM in Z direction (with respect to DTCS body axis). As the global performance index for both directions Y, Z (with respect to DTCS body axis) using TTM is much less when compared with the WPTM. On the other hand when applying the same performance index to both methods (TTM, WPTM) for yaw rate, yaw acceleration and yaw jerk, it is clear that the TTM energy consumption during the tracking maneuvers is much more than the energy consumed by the WPTM as it is shown in the following figures Fig. 34, Fig. 35 and Fig. 36, $[4,5]$.

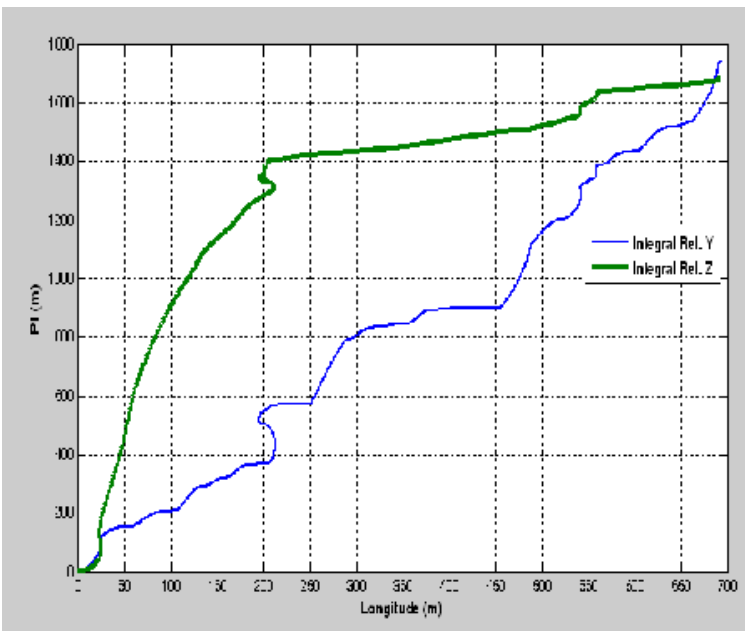

Fig. 32. Performance Index in $(Y, Z)$ Directions for TTM

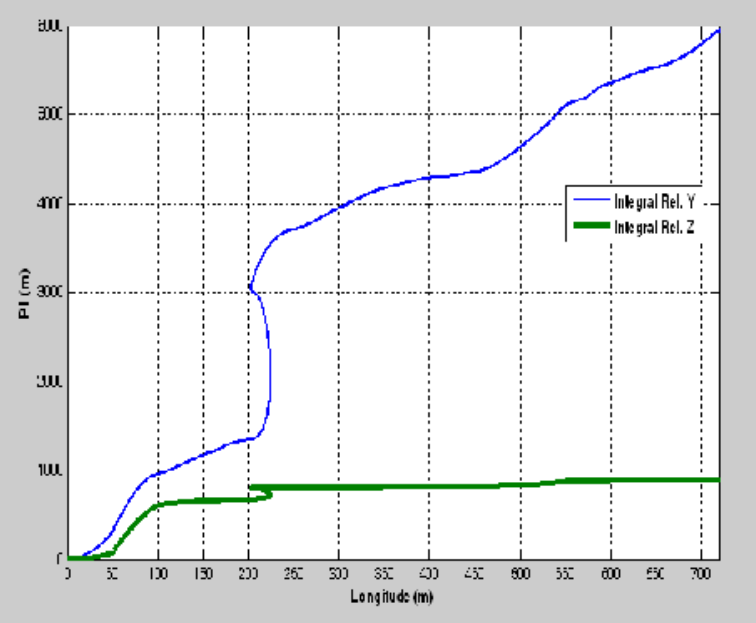

Fig. 33. Performance Index in $(Y, Z)$ Directions for WPTM 


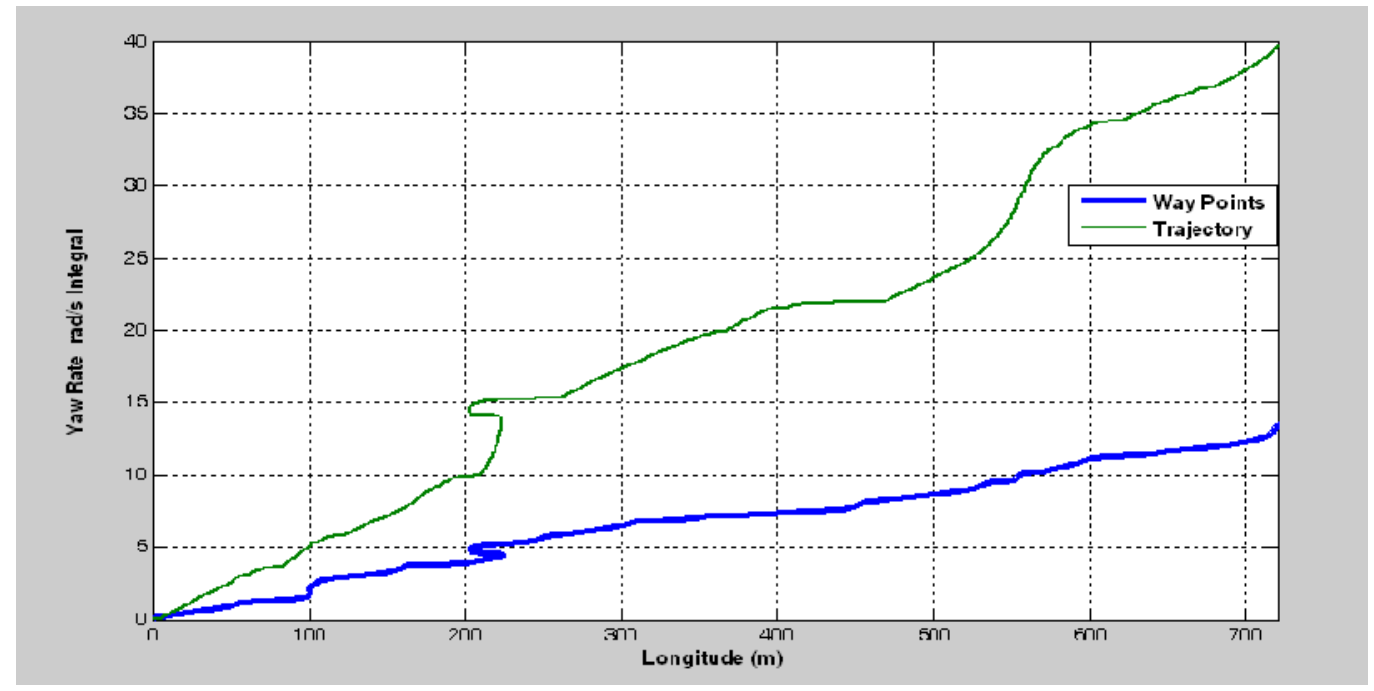

Fig. 34. Absolute Yaw Rate Integral for both TTM and WPTM

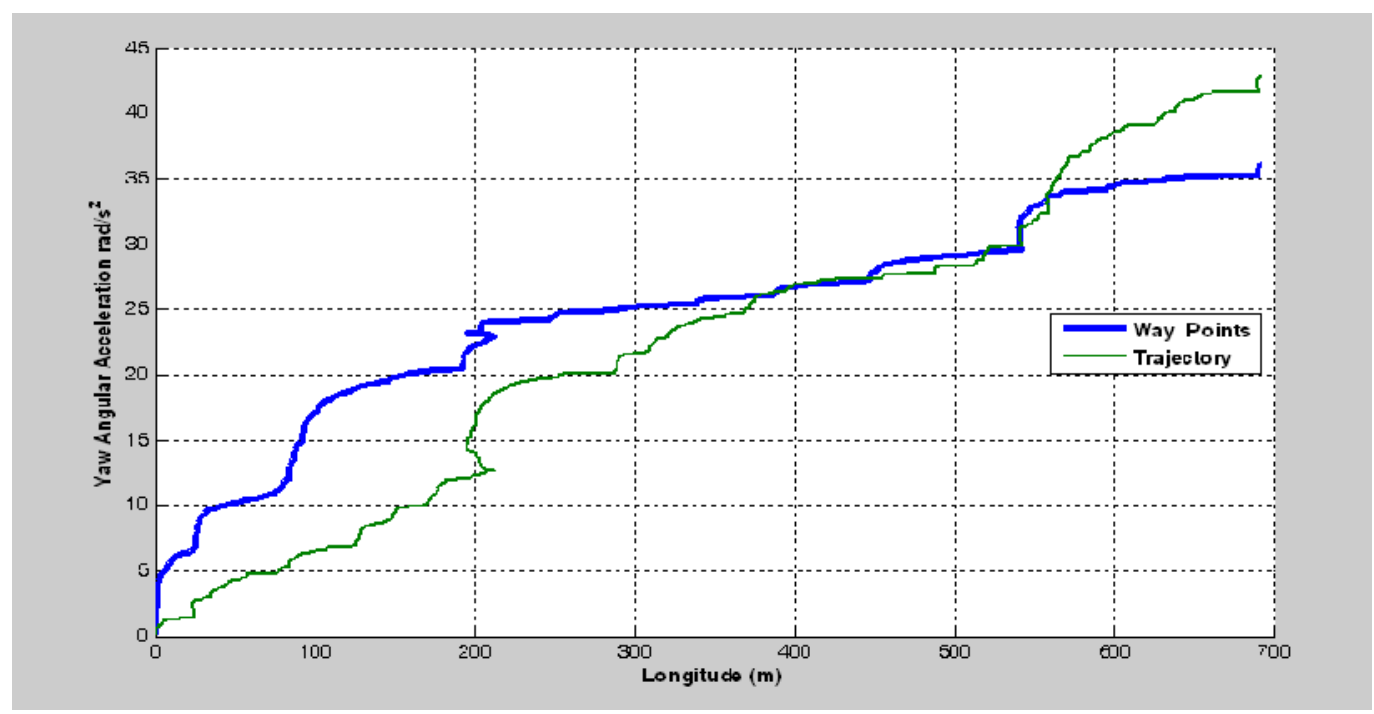

Fig. 35. Absolute Yaw Angular Acceleration Integral for both TTM and WPTM

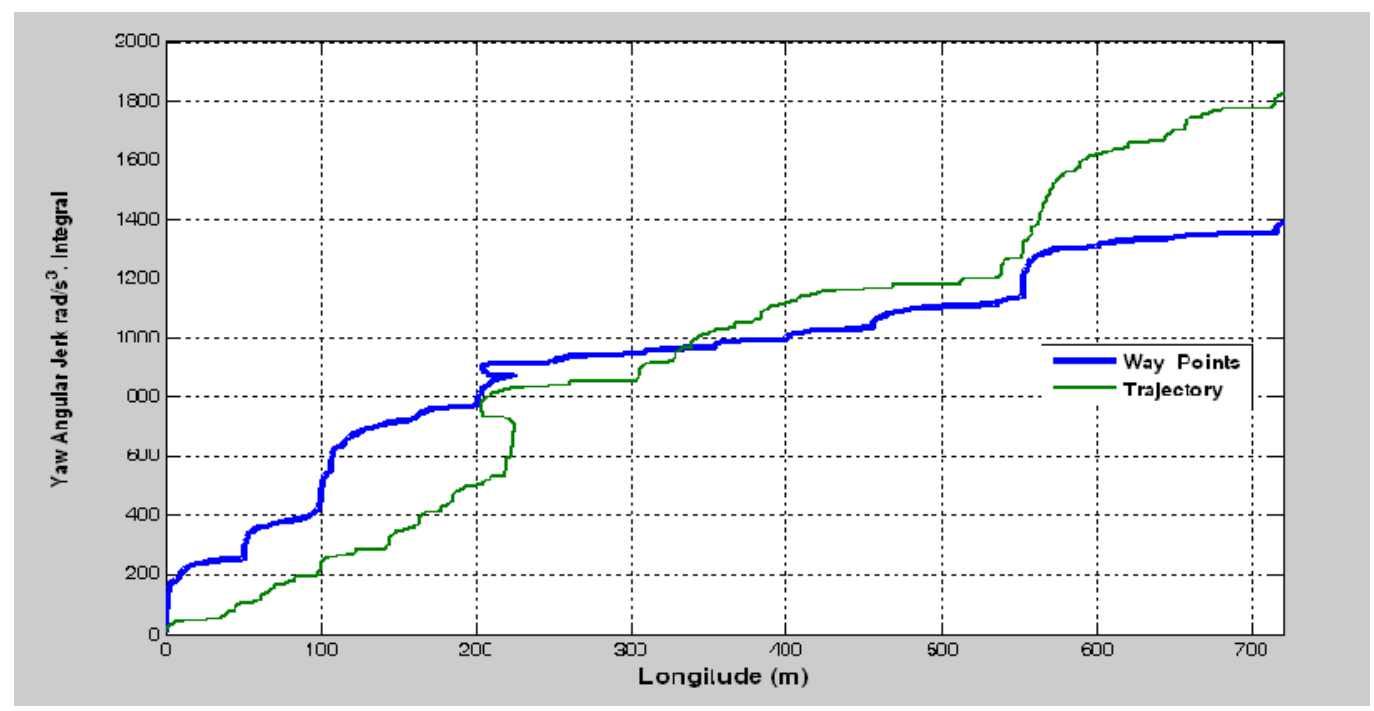

Fig. 36. Absolute Yaw Angular Jerk Integral for both TTM and WPTM 


\section{Conclusion}

In this paper it is concluded that it is recommended to use TTM in case when the unmanned flying model is required to track a planned trajectory with less relative errors, while the WPTM is recommended when the precision in tracking a planned trajectory is not an objective. However the control energy consumed by TTM is obviously more than WPTM for the same planned trajectory (Flight Path), thus the control servos using TTM would require more batteries than they would require when using WPTM. The application of hybrid system that utilizes both methods advantage is strongly recommended in this case. Using both methods will allow applying TTM during loitering when the tracking precision is required, and applying WPTM when it is only required to pass the waypoints without tracking the flight path passing those waypoints (when covering distances only is required during flight).

\section{References}

[1] Shamsudin S. S., "The Development of Autopilot System for an Unmanned Aerial Vehicle (UAV) Helicopter Model", "University of Technology, M.Sc." August, 2007, pp. 1-147.

[2] Hosny A. M., Chao H., " Development of Fuzzy Logic LQR Control Integration for Aerial Refueling Autopilot", "Proceedings of the $12^{\text {th }}$ International Conference on Aerospace Sciences and Aviation Technology, ASAT-12," May 29-31, 2007.

[3] Hosny A. M., Chao H., "Fuzzy Logic Controller Tuning Via Adaptive Genetic Algorithm Applied to Aircraft Longitudinal Motion", " Proceedings of the $12^{\text {th }}$ International Conference on Aerospace Sciences and Aviation Technology, ASAT-12," May 29-31, 2007.

[4] Hosny A. M., Chao H., "Development of A Customized Autopilot for Unmanned Helicopter Model Using Pole Placement Method Tuned By Genetic Algorithm", " Proceedings of the $12^{\text {th }}$ International Conference on Aerospace Sciences and Aviation Technology, ASAT-14," May 22-24, 2011.

[5] Goldberg, D., Genetic Algorithms in Search, Optimization \& Machine Learning, ${ }^{\text {st }}$. ed., Vol. 1, Addison Wesley Longman, 1989.

[6] Flying Qualities of Aeronautical Design Standard for military helicopter (ADS-33C) (US Army Aviation Systems Command, 1989). 